\title{
Transformation-optics insight into nonlocal effects in separated nanowires
}

\author{
A. I. Fernández-Domínguez, ${ }^{1}$ P. Zhang, ${ }^{2}$ Y. Luo, ${ }^{1}$ S. A. Maier, ${ }^{1}$ F. J. García-Vidal, ${ }^{2}$ and J. B. Pendry ${ }^{1}$ \\ ${ }^{1}$ The Blackett Laboratory, Department of Physics, Imperial College London, London SW7 2AZ, United Kingdom \\ ${ }^{2}$ Departamento de Física Teórica de la Materia Condensada, Universidad Autónoma de Madrid, E-28049 Madrid, Spain
}

(Received 19 October 2012; published 28 December 2012)

\begin{abstract}
We present a transformation-optics approach which sheds analytical insight into the impact that spatial dispersion has on the optical response of separated dimers of metallic nanowires. We show that nonlocal effects are apparent at interparticle distances one order of magnitude larger than the longitudinal plasmon decay length, which coincides with the spatial regime where electron tunneling phenomena occur. Our method also clarifies the interplay between nonlocal and radiation effects taking place in the nanostructure, yielding the dimer dimensions that optimize its light harvesting capabilities.
\end{abstract}

DOI: 10.1103/PhysRevB.86.241110

PACS number(s): 73.20.Mf, 78.67.Bf, 42.25.Bs, 42.70.-a

The impact of spatial nonlocality in the optical properties of metal nanoparticles is currently attracting great research attention. The presence of subnanometric geometric features in these nanostructures enables them to support extremely localized surface plasmon (SP) resonances. Classical electrodynamics predicts that the focusing ability of SPs is pushed to its maximum efficiency at these diminutive decorations, ${ }^{1}$ where the extent of the electromagnetic fields become comparable to the Coulomb screening length ( $\sim 0.1 \mathrm{~nm}$ for noble metals). However, the local constitutive relations of macroscopic Maxwell's equations do not reflect the occurrence of significant electron-electron interactions in this spatial regime. Thus, a nonlocal treatment of the dielectric characteristics of metals, ${ }^{2}$ beyond the free-electron Drude model, is required to clarify the limitations and guide the optimization of plasmonic devices.

Although spatial dispersion in the permittivity of metals has been intensively studied in the past, ${ }^{3-6}$ its experimental exploration has not been possible until very recently. Current fabrication and optical characterization techniques allow the probing of SP resonances below the nanometer, ${ }^{7-10}$ which has renewed the theoretical interest in the nonlocal response of metallic nanostructures. ${ }^{11-13}$ Nanoparticle dimers are probably the system most thoroughly investigated in this context. ${ }^{14-16}$ In this Rapid Communication, we revisit this geometry using a quasianalytical transformation-optics (TO) approach, ${ }^{17}$ which was first developed within the local approximation. ${ }^{18-21}$ Lately, this method has been used to describe nonlocal effects in touching nanowires. ${ }^{22}$ Here, we extend this TO framework to separated dimers, clarifying how spatial dispersion affects the light harvesting properties of these devices.

Figure 1(a) depicts a pair of metal nanowires of radius $R$ separated by a gap distance $d$, illuminated by an electric field polarized along the dimer axis. Under the logarithmic transformation indicated, the dimer maps into the metalinsulator-metal structure shown in Fig. 1(b). ${ }^{18}$ The incident electric field maps into an array of dipole sources located at $x^{\prime}=0$ with period $2 \pi$. The transformed parameters can be expressed in terms of the original ones as $g=4 R \sqrt{\rho(1+\rho)}$ and $a=2 \ln (\sqrt{\rho}+\sqrt{\rho+1})$, where $\rho=d / 4 R$ is the relative gap size. The permittivity tensor of the dimer is described using the hydrodynamical model. ${ }^{2}$ Thus, the transverse component $\epsilon_{\mathrm{T}}(\omega)=\epsilon_{\infty}\left[1-\frac{\omega_{\mathrm{P}}^{2}}{\omega(\omega+i \gamma)}\right]$ remains spatially nondispersive, whereas the longitudinal one acquires a $k$ dependence, $\epsilon_{\mathrm{L}}(\mathbf{k}, \omega)=\epsilon_{\infty}\left[1-\frac{\omega_{\mathrm{P}}^{2}}{\omega(\omega+i \gamma)-\beta^{2}|\mathbf{k}|^{2}}\right]$.

The Drude constants considered in our calculations are $\epsilon_{\infty}=1, \omega_{\mathrm{P}}=2.2 \times 10^{15} \mathrm{rad} / \mathrm{s}$, and $\gamma=1.8 \times 10^{13} \mathrm{rad} / \mathrm{s}$, which are realistic values for silver. ${ }^{23}$ Our choice of $\epsilon_{\infty}$ makes the two possible additional boundary conditions needed to describe the optical excitation of longitudinal plasmons (continuity of the normal electric field or the normal current) totally equivalent. The $\beta$ parameter is proportional to the Fermi velocity, which presents only slight variations among different noble metals. ${ }^{24}$ Thus, we take $\beta=10^{6} \mathrm{~m} / \mathrm{s}$, in agreement with

(a)

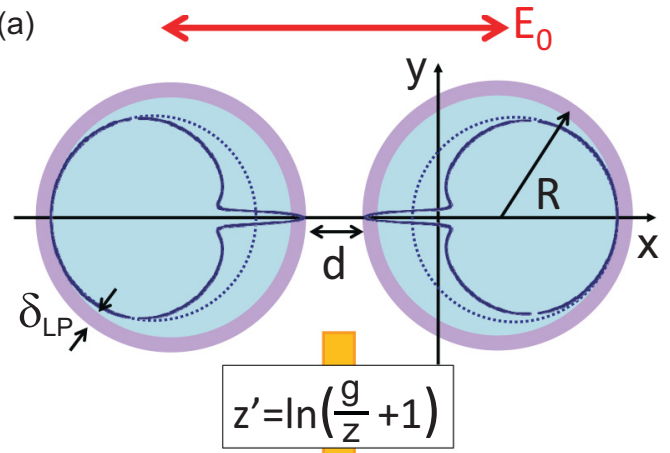

(b)

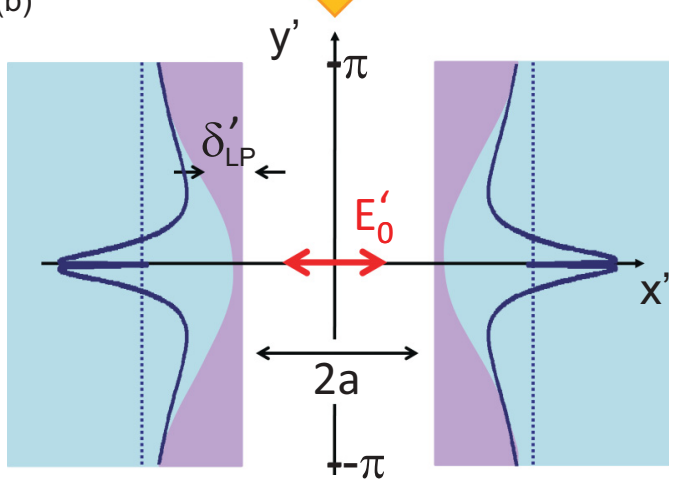

FIG. 1. (Color online) (a) Interaction of light with a dimer of separated nanowires. (b) Unit cell of the periodic structure resulting from the transformation of the original geometry. The conformal operation is shown between the two panels. The shaded areas and solid lines depict the longitudinal plasmon decay (surface charge thickness) and the surface plasmon wave function in both frames. 
a recent experimental estimation for Au nanoparticles. ${ }^{9}$ The shaded areas in Fig. 1 represent the longitudinal plasmon decay length (surface charge thickness). This is given by the constant $\delta_{\mathrm{LP}}=\beta / \omega_{\mathrm{P}}=0.45 \mathrm{~nm}$ in Fig. 1(a). The conformal operation leads to a position-dependent nonlocal parameter of the form $\beta^{\prime}\left(z^{\prime}\right)=\frac{2 \beta}{g}\left|1-\cosh z^{\prime}\right|$ in the transformed structure. This yields the surface charge thickness $\delta_{\mathrm{LP}}^{\prime}\left(y^{\prime}\right)$ outlined in Fig. 1(b). The solid lines sketch the wave function of propagating SP modes in both frames.

Maxwell's equations can be solved in the transformed geometry beyond the quasistatic approximation. ${ }^{25}$ Taking advantage of the periodic character of the structure, we reflect the excitation of longitudinal plasmons in the system through the spatial averaging of $\delta_{\mathrm{LP}}\left(y^{\prime}\right)$. Specifically, an average decay length is calculated at the vicinity of the dipole sources $\left(\left|y^{\prime}\right|<\right.$ $\pi / 2$ ) in order to describe accurately the near-field coupling of the incident fields to propagating SPs. Implementing this approximate treatment of nonlocal effects in the local solution of the problem, we can express the absorption cross section of the dimer as

$$
\sigma_{\mathrm{abs}}=\frac{(8 R)^{2} \pi \omega}{\eta^{2} c} \rho^{\prime}\left(1+\rho^{\prime}\right) \operatorname{Im}\left\{\sum_{n=1}^{\infty} \xi_{n}\right\},
$$

where

$$
\begin{gathered}
\xi_{n}=\frac{n e^{\alpha_{n}}}{\left(\sqrt{\rho^{\prime}}+\sqrt{\rho^{\prime}+1}\right)^{4 n}-e^{\alpha_{n}}}, \\
\alpha_{n}=\ln \left(\frac{\epsilon_{\mathrm{T}}(\omega)-1}{\epsilon_{\mathrm{T}}(\omega) \frac{\gamma_{n}+1}{\gamma_{n}-1}+1}\right) .
\end{gathered}
$$

Equation (3) governs the dispersion relation of the propagating SP modes at the transformed interfaces, and $\gamma_{n}=\sqrt{1+\left(\frac{2 R}{\beta}\right)^{2} \frac{\omega_{\mathrm{P}}^{2}-\omega^{2}}{n^{2}} \frac{\rho^{\prime}\left(\rho^{\prime}+1\right)}{\left(2 \rho^{\prime}+1-2 / \pi\right)^{2}}}$ is the ratio between the normal and tangential wave-vector components of the longitudinal plasmons excited in the structure. Note that in the $\beta \rightarrow 0$ limit, $\gamma_{n} \rightarrow \infty$ and the equations above recover their local form. ${ }^{18}$

The ratio $\rho^{\prime}=\rho+\rho_{0}$, where $\rho_{0}=d_{0} / 4 R$, follows from the introduction of the parameter $d_{0}$ into the theory. This sets an effective minimum gap size for the dimer, and it is found by requiring Eq. (1) to recover the TO nonlocal predictions for touching nanowires ${ }^{22}$ in the limit $d=0$. Note that the latter are obtained from the inversion of the original structure. Specifically, $d_{0}$ is calculated by matching the resonant condition in the denominator of Eq. (2) (evaluated at $n=1$ and $\rho^{\prime}=\rho_{0}$ ) to the spectral position of the first absorption maximum in the touching case. This procedure makes possible the correction of the approximate treatment of $\delta_{\mathrm{LP}}\left(y^{\prime}\right)$, which breaks down for large SP wave vectors and small relative gap sizes. Finally, the term $\eta=\left|1-2 \pi i(2 R \omega / c)^{2} \rho^{\prime}\left(1+\rho^{\prime}\right) \sum_{n=1}^{\infty} \xi_{n}\right|$ in Eq. (1) corresponds to the radiative reaction factor, ${ }^{25}$ which accounts for the appearance of radiative losses as the dimer dimensions become comparable to the incoming wavelength.

Figure 2 renders the absorption efficiency (defined as the cross section over the dimer physical size) versus normalized frequency $\left(\omega_{\mathrm{SP}}=\omega_{\mathrm{P}} / \sqrt{2}\right)$ for touching nanowires of $10 \mathrm{~nm}$ radius. The main panel shows the good agreement between the spectrum obtained from Eq. (1) (blue solid line) and the prediction from Ref. 22 (red dashed line). Note that these two

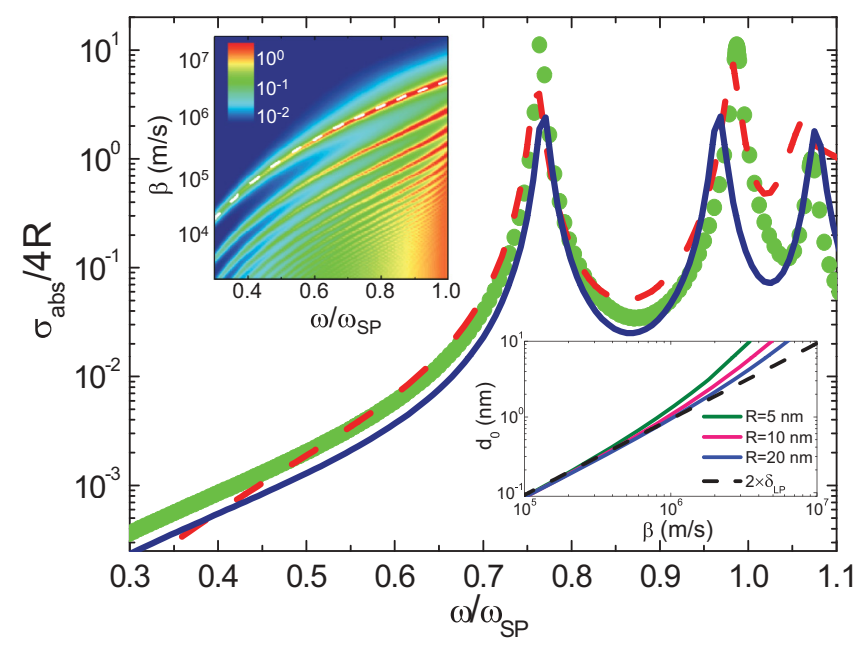

FIG. 2. (Color online) Absorption efficiency of $10 \mathrm{~nm}$ radius touching nanowires obtained under the inversion (red dashed line) and the logarithmic transformation (blue solid line) of the original dimer. For comparison, nonlocal numerical simulations are shown as green dots. The upper inset renders $\sigma_{\mathrm{abs}} / 4 R$ in $\log$ scale vs the incident frequency and $\beta$ for $R=10 \mathrm{~nm}$. The white dashed line plots the fitting to the lowest resonant frequency used to calculate $d_{0}$. The lower inset renders $d_{0}$ vs $\beta$ for three different nanowire radii, together with $2 \delta_{\mathrm{LP}}$.

sets of data were obtained under different treatments of spatial dispersion, which explains the small discrepancies between them. For comparison, nonlocal numerical simulations ${ }^{26}$ are also plotted as green dots. The upper inset displays the absorption efficiency of touching dimers $(R=10 \mathrm{~nm})$ as a function of $\omega / \omega_{\mathrm{SP}}$ and $\beta$. The white dashed line plots the resonant frequency used to calculate $d_{0}$. The lower inset renders the minimum gap size versus $\beta$ for three different nanowire radii, together with $2 \delta_{\mathrm{LP}}$. Note that $d_{0} \simeq 2 \delta_{\mathrm{LP}}$ for realistic $\beta$. This fact reveals that the physical origin of the parameter $d_{0}$ resides in the nonlocal thickening of the charge distribution at the nanowire surface, which effectively blurs the dimer boundaries. At large $\beta$ and small $R, d_{0}>2 \delta_{\mathrm{LP}}$, which indicates that spatial dispersion cannot be simply linked to the opening of an effective gap at the dimer contact. This deviation reflects that nonlocal effects alter significantly the optical properties of small $(R \lesssim 5 \mathrm{~nm})$ isolated nanowires. ${ }^{11}$

The upper panels in Fig. 3 display the absorption efficiency $\sigma_{\mathrm{abs}} /(4 R+d)$ of separated nanowires $(R=10 \mathrm{~nm})$ as a function of the incident frequency and the relative gap size $\rho$. Figure 3(a) renders local calculations, whereas Figs. 3(b) and 3(c) are evaluated at $\beta=10^{5}$ and $10^{6} \mathrm{~m} / \mathrm{s}$, respectively (the latter is a realistic estimation for noble metals). For large gaps, our theory reproduces the nonlocal blueshift experienced by the dipolar resonance of single cylinders. ${ }^{6}$ Figures 3(b) and 3(c) show that spatial dispersion truncates the continuous redshifting of the absorption maxima with decreasing $d$ obtained within the local approximation. ${ }^{18}$ Recently, this spectral deviation from the local predictions has been verified experimentally in a gold nanosphere, gold substrate configuration. ${ }^{9}$ Remarkably, Fig. 3 evidences that the impact of nonlocality in the spectral position of the SP resonances is apparent at gap sizes one order of magnitude larger than $\delta_{\mathrm{LP}} \simeq d_{0} / 2$. Note that the position of 

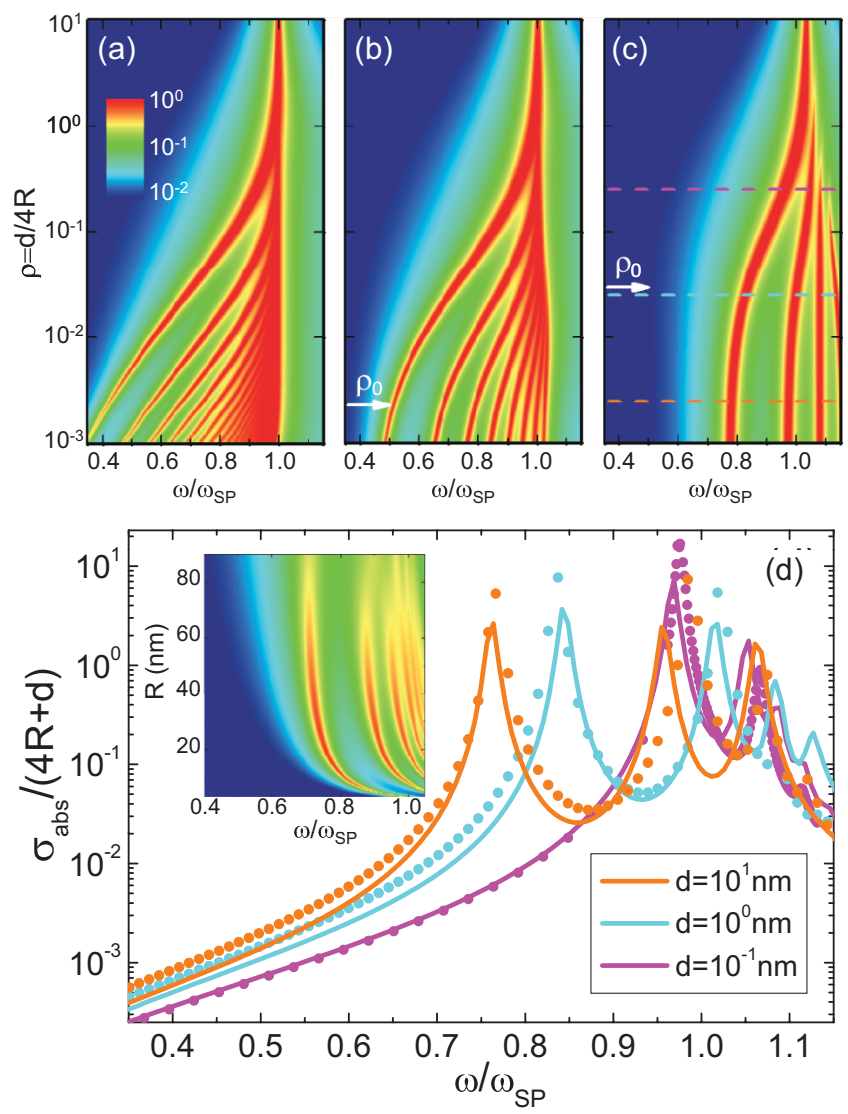

FIG. 3. (Color online) The upper panels show $\sigma_{\mathrm{abs}} /(4 R+d)$ for separated nanowires as a function of the incident frequency and the relative gap size for three different $\beta$ : (a) local, (b) $10^{5} \mathrm{~m} / \mathrm{s}$, and (c) $10^{6} \mathrm{~m} / \mathrm{s}$. (d) plots the theoretical (solid line) and numerical (dots) absorption spectra for three different gap sizes $(R=10 \mathrm{~nm})$. The inset displays $\sigma_{\mathrm{abs}} /(4 R+d)$ vs $\omega / \omega_{\mathrm{SP}}$ and $R$ for $\rho=0.025$ in the same color scale as the upper panels.

$\rho_{0}$ is marked by a white horizontal arrow in each panel. In good agreement with previous theoretical reports, ${ }^{14}$ our TO approach predicts that the intensity of the absorption maxima is rather insensitive to spatial dispersion.

Importantly, our method does not take into account the occurrence of charge transfer phenomena due to electron tunneling in plasmonic dimers. Full quantum calculations yield a further blueshift and a drastic lowering of the absorption peaks due to these effects at angstrom-sized gap distances. ${ }^{27-29}$ A recent experimental work on gold nanoparticles has set the length scale for this quantum tunneling regime at $d_{\mathrm{QR}}=$ $0.31 \mathrm{~nm}$ (Ref. 10) (note the similarity with $\delta_{\mathrm{LP}}$ above). This distance is dictated by the metal work function which, to a good approximation, is the same for all noble metals. ${ }^{24}$ Therefore, our theoretical findings indicate that significant nonlocal effects take place in silver nanowires at interparticle distances one order of magnitude larger than $d_{\mathrm{QR}}$.

Figure 3(d) plots $\sigma_{\mathrm{abs}} /(4 R+d)$ for $R=10 \mathrm{~nm}$ nanowires separated by the three relative gap sizes indicated by dashed color lines in Fig. 3(c). The good agreement between theoretical (solid lines) and numerical (dots) calculations demonstrates the validity of our TO method at $\omega \lesssim \omega_{\text {SP }}$. At higher frequencies, our approach overestimates the electromagnetic contribution of SP modes with large propagating wave vector (large $n$ ) and the comparison with numerical simulations worsens. As discussed above, this is caused by the approximate treatment of nonlocality inherent to Eq. (1). In the inset of Fig. 3(d), we explore the impact of radiation losses in the dimer performance. The panel renders the absorption efficiency versus $\omega / \omega_{\mathrm{SP}}$ and $R$ for nanowires with $\rho=0.025$ (which corresponds to $d=1 \mathrm{~nm}$ at $R=10 \mathrm{~nm}$ ). The normalized cross section is reduced considerably for $R>60 \mathrm{~nm}$ due to radiative reaction. On the other hand, spatial dispersion diminishes $\sigma_{\text {abs }} /(4 R+d)$ below $10 \mathrm{~nm}$. The interplay between these two effects leads to a range of nanowire radii around $40 \mathrm{~nm}$ where the absorption efficiency of the dimer is maximized.

So far, we have analyzed how nonlocal effects modify the light collection properties of separated dimers. In the following, we investigate how the local description of their nanofocusing performance is corrected once spatial dispersion is taken into account. With this purpose, we concentrate on a single magnitude, the absolute value of the field enhancement at the gap center. We consider only the electric field component parallel to the dimer axis ( $x$ direction), which is the largest across the gap region. Our TO method yields a very simple expression for the field enhancement defined this way,

$$
\left|\frac{E_{\text {gap }}}{E_{0}}\right|=\frac{1}{\eta}\left|1+8 \sum_{n=1}^{\infty}(-1)^{n+1} \xi_{n}\right|,
$$

where the first term reflects the contribution of the incident electric field and $\xi_{n}$ is defined in Eq. (2).

Figure 4(a) plots the field enhancement versus frequency for $10 \mathrm{~nm}$ radius nanowires separated by a $0.5 \mathrm{~nm}$ gap. Local and realistic nonlocal $\left(\beta=10^{6} \mathrm{~m} / \mathrm{s}\right)$ calculations are rendered in gray and black lines, respectively. As observed in the absorption cross section, the introduction of spatial
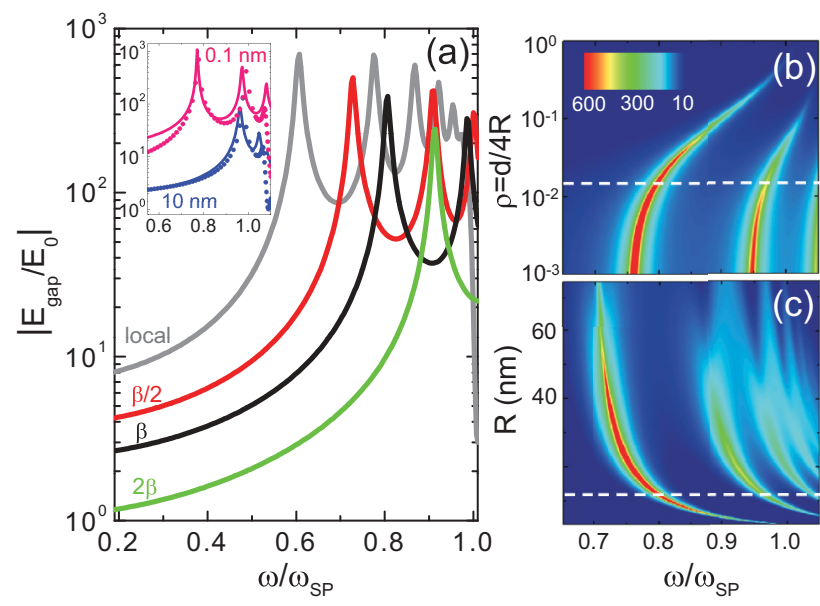

FIG. 4. (Color online) (a) Absolute value of the field enhancement at the gap center $\left|E_{\text {gap }} / E_{0}\right|$ vs frequency for $R=10 \mathrm{~nm}$ and $d=$ $0.5 \mathrm{~nm}\left(\beta=10^{6} \mathrm{~m} / \mathrm{s}\right)$. The inset shows the comparison between theory (solid lines) and simulations (dots) for $d=0.1 \mathrm{~nm}$ and $d=10$ nm. (b) $\left|E_{\text {gap }} / E_{0}\right|$ as a function of $\omega / \omega_{\mathrm{SP}}$ and $\rho$ for $R=10 \mathrm{~nm}$. (c) Field enhancement vs frequency and $R$ for nanowire dimers with $\rho=0.025$. (b) and (c) are evaluated at $\beta=10^{6} \mathrm{~nm}$. The white dashed lines in (b) and (c) correspond to the realistic nonlocal spectrum in (a). 
dispersion blueshifts and reduces the number of plasmonic resonances supported by the nanostructure below $\omega_{\mathrm{SP}}$. Figure 4(a) indicates that nonlocality lowers $\left|E_{\text {gap }} / E_{0}\right|$ not only at the spectral peaks, but also at frequencies out of resonance. However, note that the maximum enhancement (located at $0.6 \omega_{\mathrm{SP}}$ and $0.8 \omega_{\mathrm{SP}}$ in the local and nonlocal spectra, respectively) is diminished by only a factor of 2 for the geometry and metal permittivity considered here. In order to gain physical insight into this result, $\left|E_{\text {gap }} / E_{0}\right|$ for nonlocal parameters equal to $2 \beta$ and $\beta / 2$ are shown as red and green lines, respectively. These spectra demonstrate indeed that increasing $\beta$ leads to a continuous blueshift and lowering of the field enhancement resonances. The inset of Fig. 4(a) displays the comparison of our theoretical calculations with numerical simulations for two extreme configurations $(R=$ $10 \mathrm{~nm}$ ): $d=0.1 \mathrm{~nm}$ (pink) and $d=10 \mathrm{~nm}$ (blue). Whereas the agreement is very good at large $d$, the theory seems to overestimate $\left|E_{\text {gap }} / E_{0}\right|$ at low frequencies for small gap sizes. In both cases, the approximate description of spatial dispersion in Eq. (4) prevents it from reproducing the abrupt drop in the numerical field enhancement at $\omega \approx 1.1 \omega_{\text {SP }}$.

Figure 4(b) displays the field enhancement at the gap center as a function of the incident frequency and $\rho$ for $R=10 \mathrm{~nm}$. As expected, the field enhancement maxima follow the same spectral trend as the absorption peaks [see Fig. 3(c)]. Whereas the local approximation yields enormous $\left|E_{\text {gap }} / E_{0}\right|$ values for very small gaps, our TO treatment indicates that this superfocusing ability is truncated by spatial dispersion. Our nonlocal results evidence that the maximum field enhancement is below $10^{3}$, and occurs for $\rho \simeq 10 \rho_{0}$. For smaller gaps, $\left|E_{\text {gap }} / E_{0}\right|$ saturates to the touching prediction. Note that, as mentioned above, our approach does not reflect the impact of quantum tunneling for $d \leqslant d_{\mathrm{QR}}$. Finally, Fig. 4(c) analyzes the balance between radiative and nonlocal effects in the light enhancement at the gap center for dimers with $\rho=0.025$ (which corresponds to $d=1 \mathrm{~nm}$ for $R=10 \mathrm{~nm}$ ). As observed for $\sigma_{\mathrm{abs}}$, these two mechanisms lower significantly $\left|E_{\mathrm{gap}} / E_{0}\right|$ for nanowire radii larger than $50 \mathrm{~nm}$ and smaller than $10 \mathrm{~nm}$. Thus, we can conclude that both the absorption and focusing efficiency of the device are optimized for dimers with $R \sim$ $40 \mathrm{~nm}$.

In summary, we have presented a transformation-optics approach that makes possible the quasianalytical description of nonlocal effects in dimers of separated metal nanowires. We have shown that spatial dispersion blueshifts the local resonances supported by this nanostructure, in agreement with recent experimental reports. Our theoretical results indicate that this nonlocal deviation is evident for interparticle distances one order of magnitude larger than the longitudinal plasmon decay length (which coincides with the spatial regime where quantum tunneling phenomena become relevant). On the other hand, our theory predicts a relatively small lowering of the maximum absorption and field enhancement efficiencies due to nonlocality at nanometric gap sizes. Finally, the introduction of radiative losses allows us to determine that the light harvesting performance of the device is optimized for $40 \mathrm{~nm}$ radius nanowires.

This work was supported by the Leverhulme Trust, the Engineering and Physical Sciences Research Council (EPSRC), and the European Research Council (Contract No. 290981 PLASMONANOQUANTA).
${ }^{1}$ L. Novotny and B. Hech, Principles of Nano-Optics (Cambridge University Press, New York, 2008).

${ }^{2}$ A. D. Boardman, Electromagnetic Surface Modes (Wiley, New York, 1982).

${ }^{3}$ A. R. Melnyk and M. J. Harrison, Phys. Rev. B 2, 835 (1970).

${ }^{4}$ R. Fuchs and F. Claro, Phys. Rev. B 35, 3722 (1987).

${ }^{5}$ R. Ruppin, Phys. Rev. B 45, 11209 (1992).

${ }^{6}$ R. Ruppin, Opt. Commun. 190, 205 (2001).

${ }^{7}$ H. Duan, A. I. Fernández-Domínguez, M. Bosman, S. A. Maier, and J. K. W. Yang, Nano Lett. 12, 1683 (2012).

${ }^{8}$ J. A. Scholl, A. L. Koh, and J. A. Dionne, Nature (London) 483, 421 (2012).

${ }^{9}$ C. Ciraci, R. T. Hill, J. J. Mock, Y. Urzhumov, A. I. FernándezDomínguez, S. A. Maier, J. B. Pendry, A. Chilkoti, and D. R. Smith, Science 337, 1072 (2012).

${ }^{10}$ K. J. Savage, M. M. Hawkeye, R. Esteban, A. G. Borisov, J. Aizpurua, and J. J. Baumberg, Nature (London) 491, 574 (2012).

${ }^{11}$ S. Raza, G. Toscano, A.-P. Jauho, M. Wubs, and N. A. Mortensen, Phys. Rev. B 84, 121412(R) (2011).

${ }^{12}$ C. David and F. J. García de Abajo, J. Phys. Chem. C 115, 19470 (2011).

${ }^{13}$ A. Wiener, A. I. Fernández-Domínguez, A. P. Horsfield, J. B. Pendry, and S. A. Maier, Nano Lett. 12, 3308 (2012).

${ }^{14}$ F. J. García de Abajo, J. Phys. Chem. C 112, 17983 (2008).

${ }^{15}$ J. M. McMahon, S. K. Gray, and G. C. Schatz, Nano Lett. 10, 3473 (2010).
${ }^{16}$ G. Toscano, S. Raza, A.-P. Jauho, N. A. Mortensen, and M. Wubs, Opt. Express 20, 4176 (2012).

${ }^{17}$ J. B. Pendry, A. Aubry, D. R. Smith, and S. A. Maier, Science 337, 549 (2012).

${ }^{18}$ A. Aubry, D. Y. Lei, S. A. Maier, and J. B. Pendry, Phys. Rev. Lett. 105, 233901 (2010).

${ }^{19}$ A. I. Fernández-Domínguez, S. A. Maier, and J. B. Pendry, Phys. Rev. Lett. 105, 266807 (2010).

${ }^{20}$ Y. Luo, J. B. Pendry, and A. Aubry, Nano Lett. 10, 4186 (2010).

${ }^{21}$ Y. Luo, D. Y. Lei, S. A. Maier, and J. B. Pendry, Phys. Rev. Lett. 108, 023901 (2012).

${ }^{22}$ A. I. Fernández-Domínguez, A. Wiener, F. J. García-Vidal, S. A. Maier, and J. B. Pendry, Phys. Rev. Lett. 108, 106802 (2012).

${ }^{23}$ P. B. Johnson and R. Christy, Phys. Rev. B 6, 4370 (1972).

${ }^{24}$ N. W. Ashcroft and N. D. Mermin, Solid State Physics (Thomson Learning, Toronto, 1976).

${ }^{25}$ A. Aubry, D. Y. Lei, S. A. Maier, and J. B. Pendry, ACS Nano 5, 3293 (2011).

${ }^{26}$ All the simulations presented were performed using the finite element method solver in COMSOL Multiphysics.

${ }^{27}$ J. Zuloaga, E. Prodan, and P. Nordlander, Nano Lett. 9, 887 (2009).

${ }^{28}$ D. C. Marinica, A. K. Kazansky, P. Nordlander, J. Aizpurua, and A. G. Borisov, Nano Lett. 12, 1333 (2012).

${ }^{29}$ R. Esteban, A. G. Borisov, P. Nordlander, and J. Aizpurua, Nat. Commun. 3, 825 (2012). 